\title{
Florence Mourlhon-Dallies, Enseigner une langue à des fins professionnelles
}

Paris : Didier, 2008

\section{Monique Mémet}

\section{(2) OpenEdition}

\section{Journals}

Édition électronique

URL : http://journals.openedition.org/asp/72

DOI : $10.4000 / a s p .72$

ISBN : 978-2-8218-0410-4

ISSN : 2108-6354

\section{Éditeur}

Groupe d'étude et de recherche en anglais de spécialité

\section{Édition imprimée}

Date de publication : 1 novembre 2009

Pagination : 128-131

ISSN : 1246-8185

\section{Référence électronique}

Monique Mémet, «Florence Mourlhon-Dallies, Enseigner une langue à des fins professionnelles », ASp [En ligne], 56 | 2009, mis en ligne le 10 décembre 2009, consulté le 06 novembre 2020. URL : http:// journals.openedition.org/asp/72 ; DOI : https://doi.org/10.4000/asp.72

Ce document a été généré automatiquement le 6 novembre 2020 .

Tous droits réservés 
Florence Mourlhon-Dallies, Enseigner une langue à des fins professionnelles

Paris : Didier, 2008

Monique Mémet

\section{RÉFÉRENCE}

Mourlhon-Dallies Florence. 2008. Enseigner une langue à des fins professionnelles. Paris :

Didier, 351 p. ISBN 978-2278-06271-3. 
1 La langue de référence au cœur de l'ouvrage de Florence Mourlhon-Dallies, Enseigner une langue à des fins professionnelles, est le français ; cependant l'auteur s'appuie sur l'anglais de spécialité (ASP) pour l'étude de ce qu'elle dénomme le «français langue professionnelle». C'est l'une des raisons pour lesquelles est présenté dans la revue ASp cet ouvrage qui, selon la $4^{\mathrm{e}}$ de couverture, s'adresse à un public de "chercheurs en didactique des langues, enseignants de langues (pour adultes), spécialistes de l'analyse des discours professionnels, formateurs en entreprise, décideurs institutionnels et responsables de plans de formation en entreprise ».

2 Les trois parties de l'ouvrage sont divisées en onze chapitres. La première, « $\mathrm{Du}$ français, langue de spécialité au français

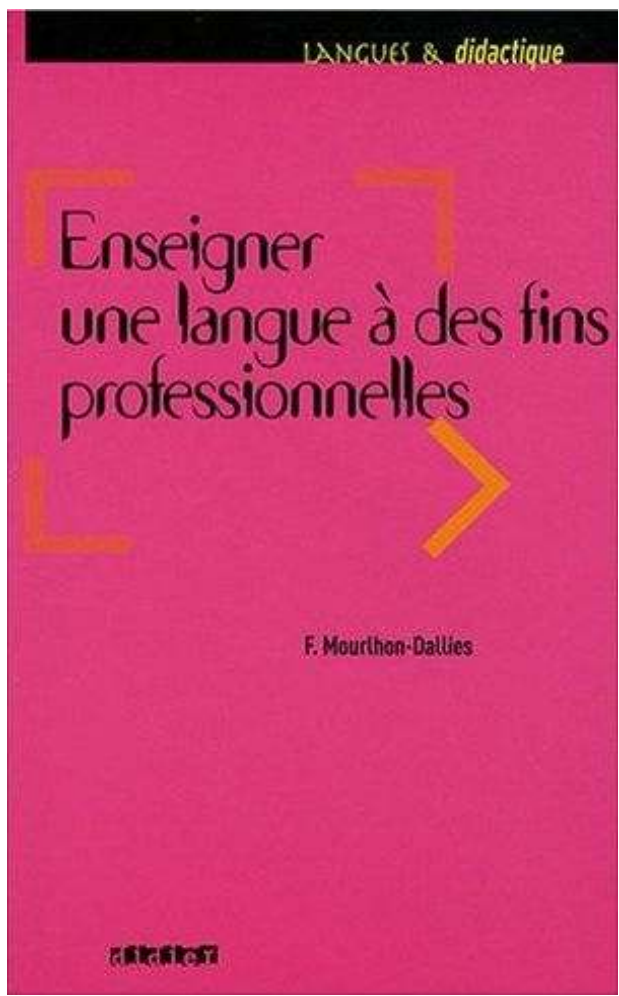
langue professionnelle» qui comprend trois chapitres, retrace l'historique de l'enseignement $d u$ français langue étrangère (FLE) à des publics professionnels. La seconde partie, en trois chapitres, traite du cadre théorique dans lequel s'inscrit cette étude fouillée ; les choix théoriques de l'auteur y sont discutés méthodiquement. Enfin, la dernière partie présente un panorama de formations à des fins professionnelles, l'«ingénierie de formation »; les cinq chapitres suivent les étapes de la conception d'un module d'enseignement.

Dans les deux premiers chapitres, F. Mourlhon-Dallies fait un rappel historique détaillé des «différents courants qui ont présidé à l'enseignement du français langue étrangère » (p. 7) en partant des expressions usitées depuis 1960, telles «vocabulaires spécialisés, français instrumental, français fonctionnel, langue de spécialité, français de la communication professionnelle... ». Elle en souligne les tendances qui se dégagent au fil des années : «le dépassement de la langue au profit du discours [et] le passage du spécialisé au professionnel»(p.47). L'auteur retrace ensuite plus longuement le français sur objectif(s) spécifique(s) (FOS, chapitre 2) ; elle en explique la spécificité et montre comment ce français sur objectifs spécifiques s'est tout d'abord décliné au pluriel. Elle fait ressortir les liens entre le FOS et l'anglais de spécialité, notamment la branche anglo-saxonne (English for Specific Purposes) alors en pleine expansion. Le FOS est passé ensuite en quelque sorte au singulier (" objectif spécifique »), en répondant aux besoins individualisés. L'auteur rappelle les démarches didactiques qui se sont succédées tout au long du $20^{\mathrm{e}}$ siècle, organisées successivement autour de la grammaire, du lexique, de la culture, de la communication et de l'action.

4 Puis, dans le troisième chapitre, l'auteur développe sa vision $d u$ «français langue professionnelle » (FLP). Celui-ci se démarque du français sur objectif spécifique en ce qu'il emprunte à d'autres disciplines que la didactique. Les publics visés diffèrent de ceux du FOS : ils exercent une profession entièrement en français et leur degré de 
professionnalisation peut varier, alors qu'en FOS les participants sont généralement déjà insérés professionnellement. Le FLP peut aussi s'appliquer à des enseignements en français langue maternelle (FLM). L'auteur présente cette forme comme «transcend[ant] donc les frontières du FLM, du FLS [français langue seconde] et du FLE » (p. 75). L'enseignement du FLP, caractérisé par la pluridisciplinarité, répond aux besoins de formation en français et dans le domaine professionnel ; il comprend trois pôles : « un pôle centré sur les aspects langagiers et linguistiques [...], un pôle activité de travail [... et] un pôle métier » (p. 81-82).

5 La deuxième partie présente le cadre théorique de l'auteur ; le lecteur suit aisément sa réflexion grâce aux arguments tour à tour avancés puis réfutés pour aboutir à la théorie propre de l'auteur. F. Mourlhon-Dallies décrit les travaux antérieurs qui ont porté sur le lien entre l'exercice de la parole et l'activité de travail, sur la "part langagière du travail » (p. 91). Elle présente les travaux des linguistes, des analystes du travail ainsi que la sociologie des entreprises. Dans le chapitre suivant (chapitre 5), elle aborde les principaux modèles d'analyse des échanges et des discours professionnels : l'« analyse des interactions au travail, l'analyse du discours et l'analyse des discours médiés ». L'auteur propose sa propre démarche qu'elle qualifie de "plus englobante et plus complexe» (p.136) et qu'elle nomme "l'Analyse Différentielle des Discours »1. Cette démarche se compose de sept étapes («hypothèses discursives, analyse du corpus de départ, résultats d'analyse, analyse comparative, bilan, modélisation de la pratique professionnelle, identification de 'logiques professionnelles'»), chaque étape faisant l'objet d'un développement argumenté.

6 Les théories sont ensuite éclairées, dans le chapitre 6, par de nombreux exemples pris dans quatre domaines très différents, ceux de l'informatique, du tourisme, de la médecine et de l'architecture. À l'intérieur de ces domaines, des comparaisons sont effectuées entre différents genres, comme les fiches pour ingénieur et pour technicien, les brochures d'agent de voyages et les dépliants d'office de tourisme, ou bien les ordonnances médicales et les notices de médicaments ou bien encore l'architecture d'intérieur et le « design d'espace ».

7 La troisième partie est en quelque sorte l'application pratique de la précédente ; il s'agit de fournir toutes les indications pour bâtir un cours en décomposant la démarche: " analyse de la demande [...], détermination des contenus à enseigner, [...] mise en œuvre de la formation et ajustements (dont évaluation et suivi)»(p. 187). Tous les aspects de chaque étape sont passés au crible des questions qui se posent lors de la création d'un module d'enseignement de FLE ou plutôt de FLP. Sont ainsi passées en revue l'analyse de la demande, l'analyse des besoins et l'analyse systémique dans le chapitre 7. Le chapitre suivant aborde la conception de la formation, en particulier les aspects financiers et juridiques, le calibrage des séances et les principes d'ordonnancement en module. Les contenus des cours et leurs objectifs sont présentés au chapitre 9 ; l'auteur dresse la liste des objectifs de contenus possibles, ceux de «type lexical», "de type grammatical», "de type phonétique/phonologique», "de type discursif », « de type juridique et déontologique », « de type socio-historique » et " de type interculturel ». Elle y ajoute la possibilité d'un huitième, "les connaissances professionnelles ». La conception d'un cours professionnel doit être affinée et c'est la raison pour laquelle l'auteur propose un chapitre pour ce qu'elle appelle "'la mise en musique' des contenus de la formation »; elle y revisite "la notion d'exercice» et y dresse une typologie des exercices possibles. Les exercices, tirés de divers manuels et 
donnés en exemple pour illustrer les phases d'élaboration d'un module de langue à des fins professionnelles, sont convaincants. Enfin le dernier chapitre traite de l'évaluation et de la certification.

8 L'ouvrage est dense et clairement structuré. Il s'appuie sur de nombreux travaux antérieurs: plus de deux cents publications figurent dans la bibliographie, qui comprend aussi 56 ouvrages de matériel pédagogique en français et en langue étrangère. Les citations sont nombreuses, traduites en français et indiquées dans la langue d'origine en notes infra-paginales; les résumés à la fin de chaque chapitre facilitent la consultation de l'ouvrage. Le lecteur regrette toutefois l'absence d'un véritable index; le livre ne comporte qu'un «index des sigles ». Même si la table des matières est très détaillée, cette dernière ne peut remplacer un index des thèmes et des auteurs.

9 La lisibilité de l'ouvrage n'est pas non plus facilitée par certains choix éditoriaux, par exemple l'absence de titre ou de numérotation pour les tableaux, la police de caractère fantaisie choisie pour les têtes de chapitres ou de sections (avec des pleins et des déliés d'épaisseur inégale), l'emploi de notes infra-paginales numérotées tout au long de l'ouvrage (il y en a 178). Les coquilles ne sont pas rares (cf. p. 52, 105, 150, 172...) et notamment en ce qui concerne l'orthographe en anglais; la mise en page pourrait être améliorée par exemple par l'ajout d'espaces insécables afin que les signes de ponctuation ne figurent pas en début de ligne, par l'absence d'usage intempestif de la majuscule, etc. Des expressions comme " penser à bout une logique» (p. 221) auraient pu être remplacées dans une publication française. Enfin, il est regrettable que la reliure d'un tel ouvrage ne résiste pas à une utilisation quelque peu intensive; les pages se détachent de la tranche au fur et mesure de la lecture.

10 Cependant les scories signalées ci-dessus sont minimes par rapport à la quantité d'informations que l'enseignant chercheur ou le formateur en langues trouvera dans l'ouvrage de F. Mourlhon-Dallies qui peut se lire d'une traite ou bien servir de livre de référence. Cet ouvrage, qui « retrace quinze années de réflexion et d'interventions de terrain » (p. 7) renouvelle le travail de fond accompli dans les années 1990 comme celui de Denis Lehmann (1993), publié dans une collection dirigée par Sophie Moirand.

11 Enseigner une langue à des fins professionnelles fourmille de données concernant la langue des milieux professionnels et l'analyse de leur discours; il sera très utile pour les enseignants chercheurs en anglais de spécialité novices qui désirent bâtir leurs cours ou retrouver l'historique de l'enseignement des langues spécialisées. Il servira également aux enseignants en ASP confirmés qui désirent réfléchir sur leurs pratiques et les remettre en question.

\section{BIBLIOGRAPHIE}

Lehmann, Denis. 1993. Objectifs spécifiques en langue étrangère ; les programmes en question. Paris : Hachette. 
NOTES

1. La capitalisation des premières lettres de très nombreux termes de l'ouvrage paraît excessive et injustifiée.

\section{AUTEURS}

\section{MONIQUE MÉMET}

École Normale Supérieure de Cachan 